Article

\title{
A Novel Magnetic Coupling for Miniaturized Bandpass Filters in Embedded Coaxial SIW
}

\author{
Stefano Sirci ${ }^{1, *(\mathbb{D}}$, Jorge D. Martínez ${ }^{2}(\mathbb{D})$ and Vicente E. Boria ${ }^{1}(\mathbb{D}$ \\ 1 iTEAM, Universitat Politècnica de València (UPV), 46022 Valencia, Spain; vboria@dcom.upv.es \\ 2 I3M, Universitat Politècnica de València (UPV), 46022 Valencia, Spain; jdmartinez@eln.upv.es \\ * Correspondence: ssirci@iteam.upv.es; Tel.: +34-96-387-7007 (ext. 88278)
}

Received: 20 December 2018; Accepted: 21 January 2019; Published: 24 January 2019

\begin{abstract}
In this paper, embedded coaxial substrate integrated waveguide (CSIW) filters with innovative magnetic couplings are presented and studied. By creating the loading capacitance of a combline topology using inner layers of a low-temperature co-fired ceramic (LTCC) stack-up, it is possible to achieve resonator miniaturization while improving the spurious-free band and providing full-packaged solutions. Moreover, a new magnetic coupling scheme consisting of short-ended stripline probes is proposed and analyzed in detail, both for direct and external couplings. An in-line three-pole filter at L-band is designed, manufactured, and measured proving how the proposed approach can be used for designing wideband bandpass filter (BPF) with extremely compact size. The designed BPF is centered at $1.5 \mathrm{GHz}$ with $10 \%$ fractional bandwidth (FBW), while the layout size is just $35 \times 9.5 \mathrm{~mm}^{2}$. The experimental results validate the coaxial SIW technology that allows for, at the same time, easy integration, compact size, flexible design, and enhanced stop-band performance.
\end{abstract}

Keywords: bandpass filters; coaxial-cavity filters; planar circuit integration; substrate integrated waveguide

\section{Introduction}

Presently, substrate integrated waveguide (SIW) technology is considered to be a fruitful approach for the design and implementation of microwave and mm-wave components, such as cavity filters, antennas, directional couplers, oscillators, or power amplifiers among others [1]. The fusion of planar and non-planar structures enabled by SIW technology presents an optimal compromise in terms of EM performance, cost, size, and manufacturability. Indeed, moderate-to-high quality factor $(Q)$, excellent planar integration, low weight, and low-cost manufacturing processes in printed circuit board (PCB) and LTCC technology are some of the key advantages that can be obtained conveniently in SIW technology.

Nevertheless, wireless communication systems are imposing increasingly stringent requirements for the performance of microwave filters to be employed for the next-generation wireless and mobile applications. In this context, compactness represents a crucial feature to be addressed in the future microwave BPFs, and for this reason, considerable effort has been recently deployed to increase the miniaturization of SIW filters whose footprint is considered to be still rather large.

As is well known, the combline topology, which can be modeled as a TEM-mode transmission line short-circuited at one end and terminated with a capacitor on the other, has been widely applied in waveguide and planar technologies for the compact implementation of high-order BPFs [2,3]. Recently, such a scheme has been successfully introduced in SIW technology by using LTCC $[4,5]$ and PCB [6] manufacturing processes, showing remarkable advantages in terms of size and EM performance with respect to standard SIW implementations. The latter are usually based on $\mathrm{TE}_{101}$-mode cavities or on more compact alternatives by bisecting the SIW resonator at quasi-perfect magnetic walls $[7,8]$. These are defined as half-mode SIW (HMSIW) and quarter-mode SIW (QMSIW) topologies, and usually 
experience increased radiation losses. For instance, in [8], an ultra-miniature two-pole filter based on eight-mode SIW resonators loaded with circular-ring miniaturized element (CRME) structures was designed in UHF band showing FBW $=10 \%$ and good spurious-free band. However, the extracted quality factor $(Q)$ of the filter was just 90 , which is comparable to lumped element implementations.

On the one hand, in $[6,9]$, the authors proposed to implement the loading capacitance of the coaxial topology by means of an isolating air gap etched on the external top metal layer of a SIW cavity resonator. This solution enables component fabrication to be fully compatible with single-layer low-cost PCB fabrication. Specifically, in [6] the feasibility of the CSIW topology was proved by designing and testing an X-band resonator. Furthermore, an X-band three-pole narrow-band BPF was designed, fabricated, and tested showing good miniaturization degree and spurious-free band performance in [9].

On the other hand, by using a multi-layer process, embedded CSIW resonators and filters were first proposed in [4] and then demonstrated in $[5,10,11]$ at lower frequency bands, such as L-band. In particular, two-pole and three-pole narrow-band BPFs were designed for UMTS/LTE-2100 applications (i.e., 2110-2170 MHz), being characterized for an improved compactness. Such a result has been achieved by implementing very high embedded coaxial loading capacitances between inner layers of a multi-layer LTCC stack-up. This topology has been recently implemented by using the PCB manufacturing in [12,13] for the design of fourth-order narrow-band BPF in X- and L-band, respectively, showing a good compromise between compactness and loss.

This work deals with the design of embedded CSIW filters in multi-layer LTCC technology with widened filtering responses, which means overcoming a typical problem of combline filters implemented in SIW technology. To do that, a novel magnetic coupling structure is proposed and studied in detail to demonstrate the feasibility of implementing such wideband filtering responses, while keeping a high miniaturization degree and a fully packaged solution. Thus, the use of the LTCC technology enables a multi-layer implementation of the filter couplings, as well as the deployment of bigger embedded loading capacitances, achieving improved performance if compared to conventional magnetic irises.

\section{Materials and Methods}

The layout and the main design parameters of an embedded CSIW resonator are shown in Figure 1. Such a structure consists of a square or circular SIW cavity where several plated via holes have been inserted at the center of the cavity resonator, as was first introduced in [4], and then demonstrated in $[5,6]$. By connecting one end of it via holes to ground, the inductive section of a coaxial resonator is created and controlled by adjusting the position and the diameter $d_{h}$ of the via holes. Conversely, at the other end of the via holes, a circular metal plate with radius $r_{p}$ is introduced, as shown in Figure $1 b$. Such a metal plate is located right below the external top metal layer, which is basically a ground plane, creating a small separating gap filled with dielectric material that we named $g_{p}$ (visible in Figure 1c).

By doing that, the strong electric field across the gap $g_{p}$ creates the integrated $C_{l}$ of a typical combline configuration. This topology enables the implementation of extremely high loading capacitances $C_{l}$ towards ground, because it is possible to increase the disk plate area considerably while keeping $g_{p}$ as narrow as possible. The capacitive component of the CSIW can be clearly seen by observing the field line distribution shown in Figure 2. It is worth mentioning that in the selected LTCC fabrication process, $g_{p}$ depends on the number and thickness of the dielectric tape layers that separate the circular disk plate from the top ground plane. 


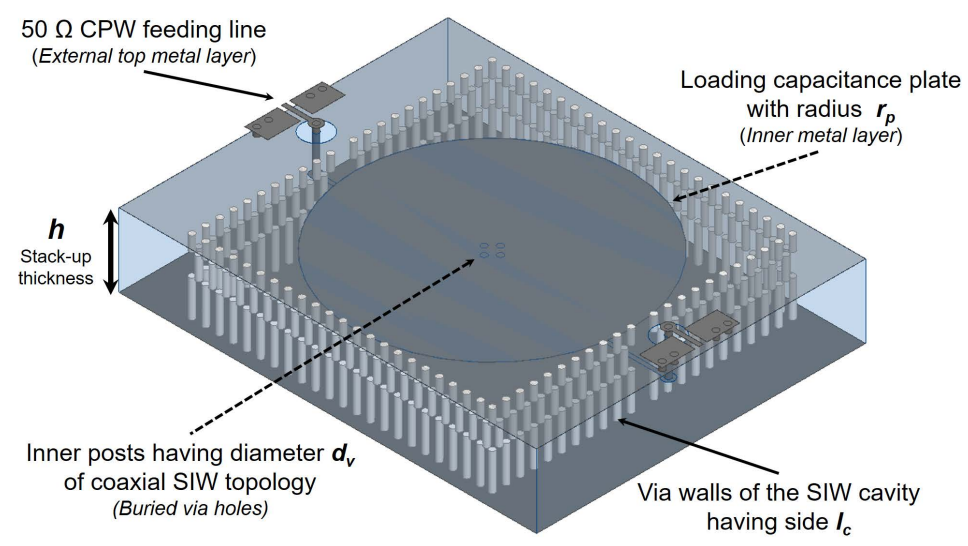

(a)

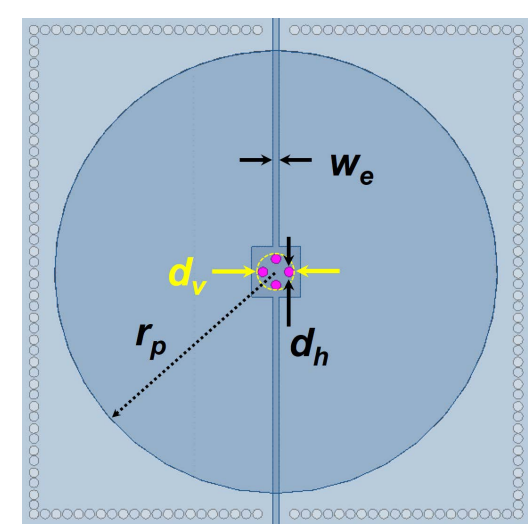

(b)

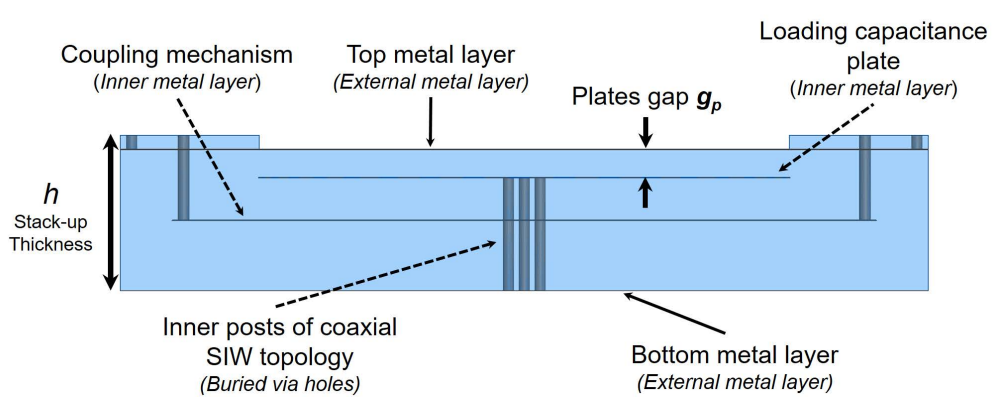

(c)

Figure 1. 3D sketches of an embedded CSIW resonator including main dimensions: (a) top view; (b) detail of the inner conductor and capacitive plate; and (c) lateral view.
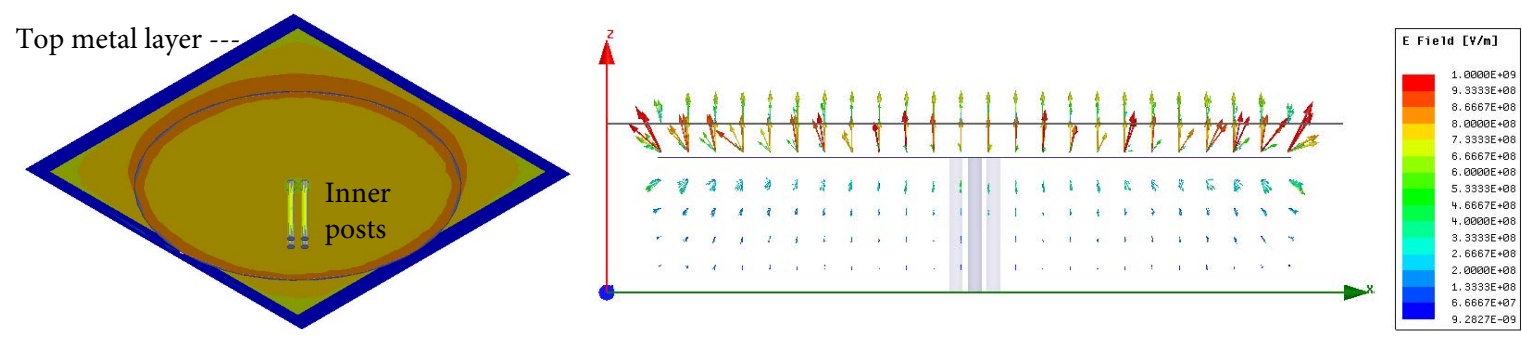

Figure 2. 3D E-field distribution for the proposed embedded coaxial SIW resonator in the gap that separates the disk plate and the top ground plane.

In addition, the electrical length of the coaxial transmission line embedded into the substrate depends on the final stack-up thickness, thus becoming an important design parameter since it controls both resonant frequency and unloaded $Q\left(Q_{u}\right)$ of the CSIW resonator [5]. Finally, the inner via holes with diameter $d_{h}$ have been symmetrically placed around the cavity center to emulate a wider circular inner conductor that would show a fictitious diameter named $d_{v}$ (see Figure $1 \mathrm{~b}$ ). The further apart the via holes are, the larger the diameter $d_{v}$. This enables us to finely set $d_{v}$ by using only the recommended value for $d_{h}$ that, in the LTCC manufacturing, should be similar to the dielectric tape thickness to enable a good via filling process.

\subsection{Synthesis}

As proposed in $[9,14]$ for the CSIW topology with a loading capacitance created by an air isolating gap, the resonator main parameters can be extracted straightforwardly, considering it as a piece of circular-square coaxial line of length $h_{p}$ (where $h_{p}=h-g_{p}$ ) and characteristic admittance $Y_{0}$ embedded 
into the dielectric substrate. Then, such elements can be modeled as a TEM-mode transmission line short-circuited at one end and terminated with a capacitor on the other. Therefore, the TEM-mode resonant frequency is given by the condition $B(\omega)=0$, being the susceptance $B$ of the coaxial resonator defined as

$$
B(\omega)=\omega C_{l}-\frac{1}{Z_{0}} \cot \beta h_{p}
$$

where $\beta$ is the TEM-mode propagation constant, and $Z_{0}$ is the coaxial resonator characteristic impedance. It should be noted that the resonator electrical length $\theta_{0}$ at the desired working frequency $f_{0}$ corresponds to $\theta_{0}=\beta h_{p}$.

Next, the characteristic impedance $Z_{0}$ of the CSIW resonator, and the proportion between the SIW cavity side $l_{c}$ and $d_{v}$ value are obtained from [14] as

$$
\begin{gathered}
Z_{0}=\left(\frac{\cot \theta_{0}+\csc ^{2} \theta_{0}}{2 b}\right) \\
\frac{l_{c}}{d_{v}}=0.9268 e^{\frac{z_{0} \sqrt{{ }_{r}}}{60}}
\end{gathered}
$$

Thus, the synthesis procedure proceeds by setting the resonator slope parameter $b$ at center frequency $\omega_{0}$ of the desired filtering response. As has been explained in [15], the susceptance slope parameter $b$ for resonators with zero susceptance at $\omega_{0}$ is well approximated by

$$
b=\frac{1}{2}\left[\omega_{0} C_{l}+\frac{\theta_{0}}{Z_{0} \sin ^{2} \theta_{0}}\right]
$$

On the one hand, the coaxial line admittance $Y_{0}$ can be calculated by using the approximation proposed in [16] for a circular inner conductor of diameter $d_{v}$ and an external square contour of side $l_{c}$, which is

$$
Y_{0}=\left[\frac{60}{\sqrt{\epsilon_{r}}} \ln \left(1.079 \frac{l_{c}}{d_{v}}\right)\right]^{-1} \text { for } l_{c} \gg d_{v}
$$

where $\epsilon_{r}$ is the dielectric substrate permittivity of the LTCC dielectric tape.

On the other hand, the loading capacitance $C_{l}$ between the circular disk and the top metal layer may be written as

$$
C_{l}=C_{p p}+\frac{C_{f f}}{1.65}=\epsilon_{r} \epsilon_{0} \frac{\pi r_{p}^{2}}{g_{p}}+\frac{C_{f f}}{1.65}
$$

where $\epsilon_{0}$ is the permittivity of the free space, $C_{p p}$ is the associated parallel plate capacitance, and $C_{f f}$ is the capacitance due to the fringing effect of the field between the edges of the plates, which are visible in the right part of Figure 2. A correcting factor has been taken into account, due to the specific configuration of the implemented parallel plate capacitor of the CSIW resonator that shows only one circular disk plate. Indeed, the other plate consists of the wider top ground plate. Thus, as demonstrated in [17], the fringing effect of a parallel plate capacitor composed of two identical circular disks can be well approximated as

$$
C_{f f}=\epsilon_{r} \epsilon_{0} r_{p} \log \left(16 \pi \frac{r_{p}}{g_{p}}-1\right)
$$

Finally, to finely tune $C_{l}$, a quick optimization of the circular disk plate radius $r_{p}$ by means of 3D EM simulations is usually required, which includes the metal layer thickness $t$ of the conducting layer that forms the plate. 


\subsection{Resonator $3 D$ EM Simulations}

To perform the study of the performance of a CSIW resonator, let us consider a benchmark configuration with center frequency $1.5 \mathrm{GHz}$ and using a 10-layer LTCC stack-up, whose layout has been already shown in Figure 1. Regarding the dielectric material, the DuPont ${ }^{\mathrm{TM}}$ Green Tape $^{\mathrm{TM}} 9 \mathrm{~K} 7$ substrate $\left(\epsilon_{r}=7.1 \pm 0.2, \tan \delta=0.0009 @ 10 \mathrm{GHz}\right)$ has been chosen due to its very low loss tangent. Each dielectric tape layer has a thickness of $0.254 \mathrm{~mm}$ in the green state, while the fired thickness is $0.224 \mathrm{~mm}$ on account of the shrinkage effect in the $z$-direction after the LTCC firing step. This means that the total thickness of the 10-layer stack-up of the CSIW resonator is $2.24 \mathrm{~mm}$.

Since we wanted to boost the miniaturization of the device with respect to standard SIW structures, we set a high value for the slope parameter, which is $b=105.5 \mathrm{mS}$. As a result, the main parameters of the CSIW resonator become: $C_{l}=10.75 \mathrm{pF}$ and $Z_{0}=61.1 \Omega$. The main dimensions of the compact CSIW resonator considered in this study are shown in Table 1 , where $p_{d}$ is the pitch between adjacent via holes forming the cavity sides. It is worth mentioning that the value of the capacitor plate separation (i.e., $g_{p}=0.448 \mathrm{~mm}$ ) has been achieved by introducing the circular disk plate after two dielectric tape layers below the external top metal layer in the stack-up. Thus, the length of the embedded coaxial line is $h_{p}=(2.24 \mathrm{~mm}-2 \cdot 0.224 \mathrm{~mm}) \simeq 1.792 \mathrm{~mm}$, which corresponds to an electrical length of $\theta_{0}=8.8^{\circ} @$ $1.5 \mathrm{GHz}$.

Table 1. Layout Dimensions of the Designed CSIW Resonator (Unit: mm).

\begin{tabular}{ccccccccc}
\hline$l_{c}$ & $h$ & $h_{p}$ & $d_{v}$ & $d_{h}$ & $r_{p}$ & $g_{p}$ & $w_{e}$ & $p_{d}$ \\
\hline 9.2 & 2.24 & 1.792 & 0.68 & 0.18 & 4.2 & 0.448 & 0.15 & 0.4 \\
\hline
\end{tabular}

These values emphasize the remarkable degree of miniaturization that can be simply achievable with the CSIW topology. Indeed, the SIW cavity side is just $9.2 \times 9.2 \mathrm{~mm}^{2}$, which corresponds to $0.126 \times 0.126 \lambda_{g}^{2}$ and $0.047 \times 0.047 \lambda_{0}^{2}$, where $\lambda_{g}$ is the guided wavelength at $1.5 \mathrm{GHz}$.

The simulated frequency response of the designed device, which has been obtained by using ANSYS HFSS software, is depicted in Figure 3a, and it is compared to the frequency response of a standard $\mathrm{TE}_{101}$-based SIW resonator having the same cavity size in Figure $3 \mathrm{~b}$. As shown, the use of an embedded CSIW topology enables also the shifting down of the fundamental mode of the SIW cavity resonator (i.e., the TEM mode in a CSIW resonator) with respect to the first spurious mode, which is the $\mathrm{TE}_{101}$ mode, thus widening the stop-band bandwidth. Indeed, the EM field of the first spurious mode is altered due to the inner via holes and capacitive patch that provoke an increase of its resonant frequency, from $8.79 \mathrm{GHz}$ as fundamental mode of a $\mathrm{TE}_{101}$-based resonator to $11.6 \mathrm{GHz}$ as first spurious mode of the CSIW resonator. Thus, in the proposed simulated CSIW structure, the stop-band bandwidth is greater than $7 \times f_{0}$, as can be seen in Figure $3 b$.

Furthermore, Figure 4 shows how the resonant frequency $\left(f_{r}\right)$ and $Q_{u}$ of the CSIW resonator change as the function of the radius $r_{p}$ of the capacitive disk, which means modifying the total $C_{l}$ value. It is evident that the resonant frequency can be controlled over a wide frequency band (i.e., $>1.5 \mathrm{GHz}$ ) without affecting the overall resonator dimensions, while keeping a constant $Q_{u}$ over frequency. 


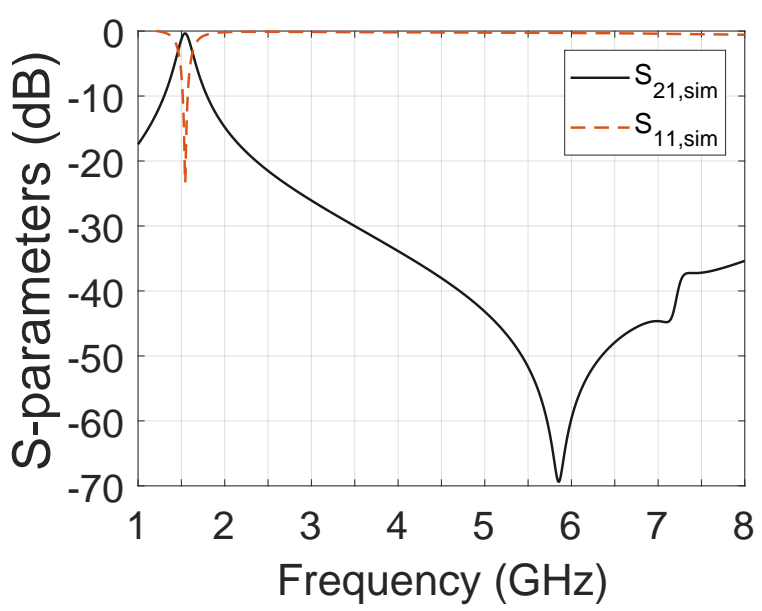

(a)

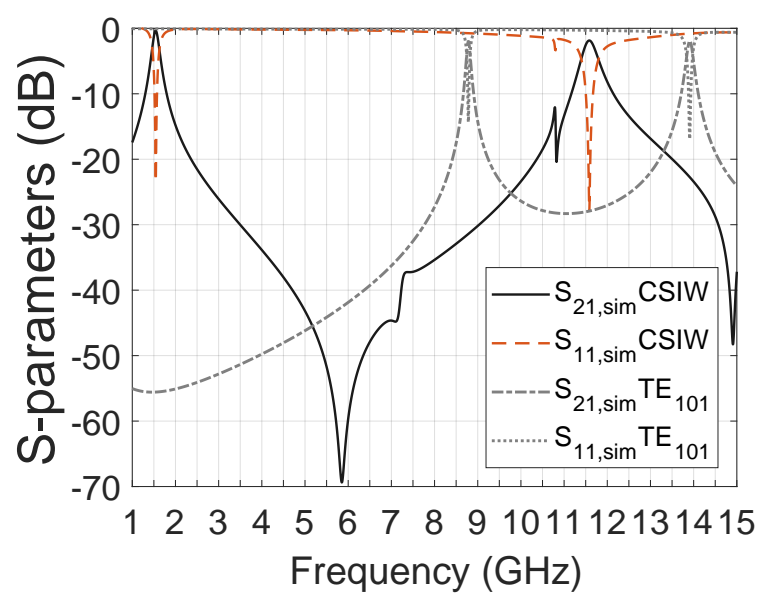

(b)

Figure 3. Simulated wideband response of (a) the proposed CSIW resonator having $b=105.5 \mathrm{mS}$; (b) Comparison between the frequency response of the proposed CSIW resonator (solid lines) and a standard $\mathrm{TE}_{101}$-based SIW resonator (dashed lines) having the same cavity size.

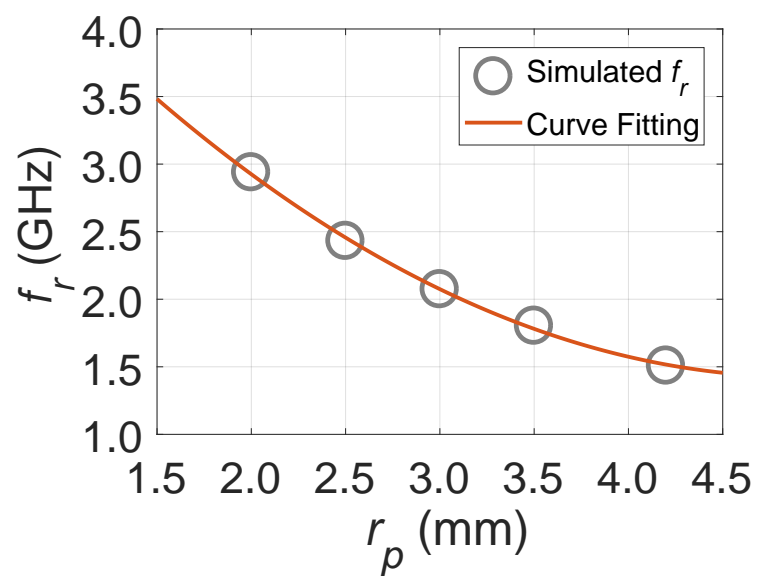

(a)

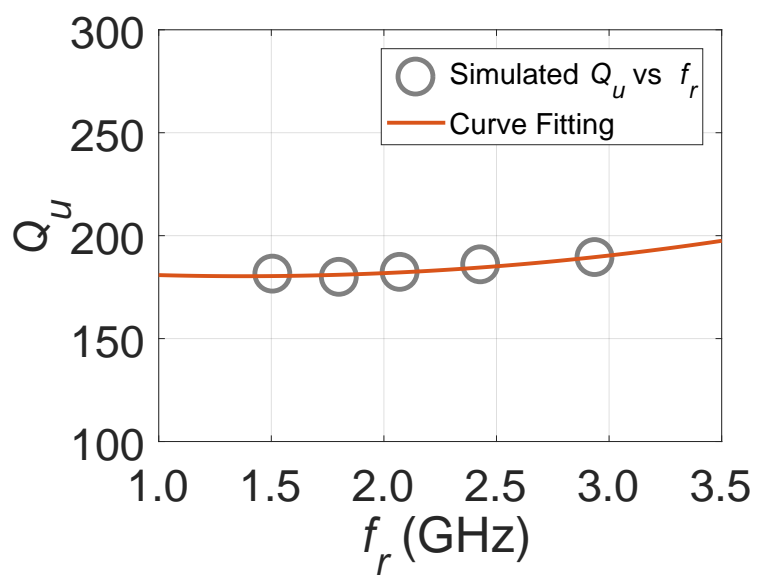

(b)

Figure 4. (a) Resonant frequency $f_{r}$ versus circular disk plate radius $r_{p}$; (b) Resonator $Q_{u}$ versus $f_{r}$ for the same variation of $r_{p}$ of (a).

In this context, Table 2 provides the comparison between $Q_{u}$, area miniaturization factor (MF, which has been defined in [18]) and spurious-free band of the designed embedded CSIW resonator and standard $\mathrm{TE}_{101}$-based SIW cavity resonators, centered also at $1.5 \mathrm{GHz}$ and implemented in the very same LTCC stack-up.

Specifically, the MF for a particular miniaturized resonator operating at $f_{0}$ of is calculated using

$$
\operatorname{MF}(\%)=\frac{\mathrm{A}_{\mathrm{SIW}, f_{0}}-\mathrm{A}_{r}}{\mathrm{~A}_{\mathrm{SIW}, f_{0}}} \times 100
$$

where $\mathrm{A}_{\mathrm{SIW}, f_{0}}$ is the area of a standard $\mathrm{TE}_{101} \mathrm{SIW}$ resonator centered at $f_{0}$ and $\mathrm{A}_{r}$ is the area of the proposed CSIW resonator.

Please note that two thickness layers have been considered for the standard SIW implementation to evaluate the effect on the $Q_{u}$. As shown in Table 2, CSIW topology enables extremely high miniaturization of the resonator with moderate degradation of $Q_{u}$ and improved stop-band performance. 
Table 2. Comparison of coaxial SIW topology versus standard TE 101 -based SIW topology.

\begin{tabular}{cccccc}
\hline SIW Topology & Area $\left(\mathbf{m m}^{2}\right)$ & MF $(\%)$ & $Q_{u}$ & $\Delta \boldsymbol{Q}_{\boldsymbol{u}}(\%)$ & Spurious-Free Band \\
\hline $\mathrm{TE}_{101}(h=2.25 \mathrm{~mm})$ & 2704 & $R E F$ & 253 & $R E F$ & $1.58 \times f_{0}$ \\
$\mathrm{TE}_{101}(h=1.12 \mathrm{~mm})$ & 2704 & 0 & 213 & -15.8 & $1.58 \times f_{0}$ \\
Coaxial $b=0.1055$ & 84.6 & 96.8 & 209 & -17.4 & $7.48 \times f_{0}$ \\
\hline
\end{tabular}

\subsection{Coupling Mechanisms}

Figure 5 depicts the topology of the novel magnetic coupling mechanism that is especially suited for obtaining strong coupling values in highly loaded CSIW resonators. Specifically, Figure 5a shows the layout of the magnetic external coupling mechanism, while the inter-resonator coupling mechanism is shown in Figure $5 \mathrm{~b}$. As reflected, the magnetic couplings are based on embedded stripline probes that are arranged at different layers of the LTCC stack-up, so that inner conductors of adjacent CSIW resonators can be short-circuited between them in a similar manner to conventional tapped-line couplings widely used in combline planar filters. Thus, this solution exploits the high flexibility of the LTCC multi-layer technology to improve the performance of CSIW filters.

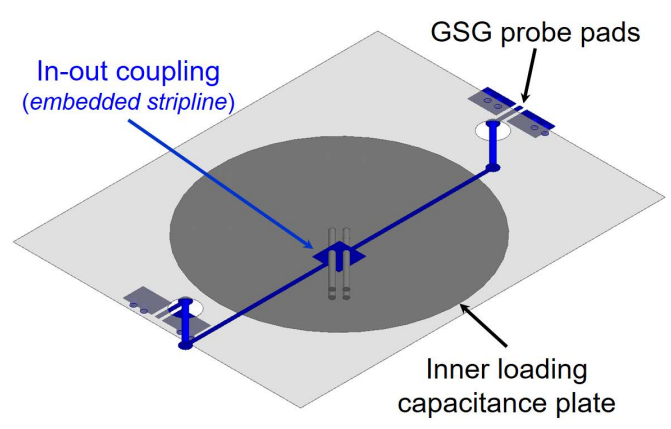

(a)

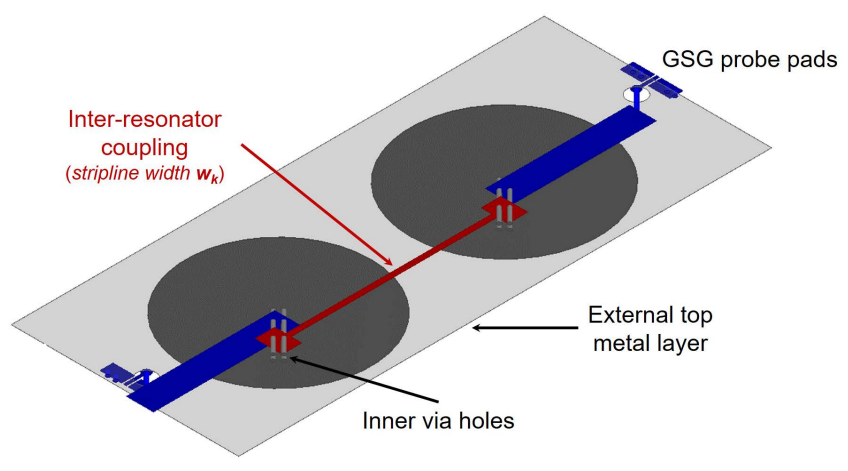

(b)

Figure 5. Layout of the proposed magnetic (a) external and (b) inter-resonator coupling mechanisms based on stripline probes short-circuited to the inner posts of the CSIW resonators. Please note that bottom layer and via holes that form the SIW cavity walls are not shown for improving readability.

By changing the height of the interconnection between coupling probes and the plated via holes forming the inner conductor, the coupling magnitude can be coarsely tuned, as shown in Figure 6 . In this context, let us name the height position in the stack-up of the external and the inter-resonator couplings as $h_{e}$ and $h_{k}$, respectively. In addition, let us set $h_{i}=0 \mathrm{~mm}$ at the external bottom level and $h_{i}=2.24 \mathrm{~mm}$ at the external top level, for $i=(e, k)$.

In Figure $6 \mathrm{a}$, the external quality factor $\left(Q_{e}\right)$ is represented as functions of $h_{e}$, while the inter-resonator coupling coefficient $\left(k_{1,2}\right)$ between two adjacent resonators versus $h_{k}$ is shown in Figure $6 \mathrm{~b}$. Taking into account that the height position of the circular disk plate corresponds to $h_{p}=1.792 \mathrm{~mm}$, the highest coupling value is achieved when the stripline probes are short-circuited to this capacitive plate element. By contrast, the coupling drops quickly as the probe is moved towards the bottom layer. Please note that such coupling values enable the design of wideband BPF with FBW easily higher than $15 \%$.

Nevertheless, a fine tuning of both couplings is achieved by varying the stripline probe width, which are named $w_{e}$ for external coupling and $w_{k}$ for inter-resonator coupling, as it can be seen in Figure $7 \mathrm{a}, \mathrm{b}$, respectively. Thus, once the height position of the coupling probes is defined in the LTCC stack-up, the probe widths allow us to finely control $Q_{e}$ and $k_{1,2}$ values, enabling a good optimization of the filtering response. 


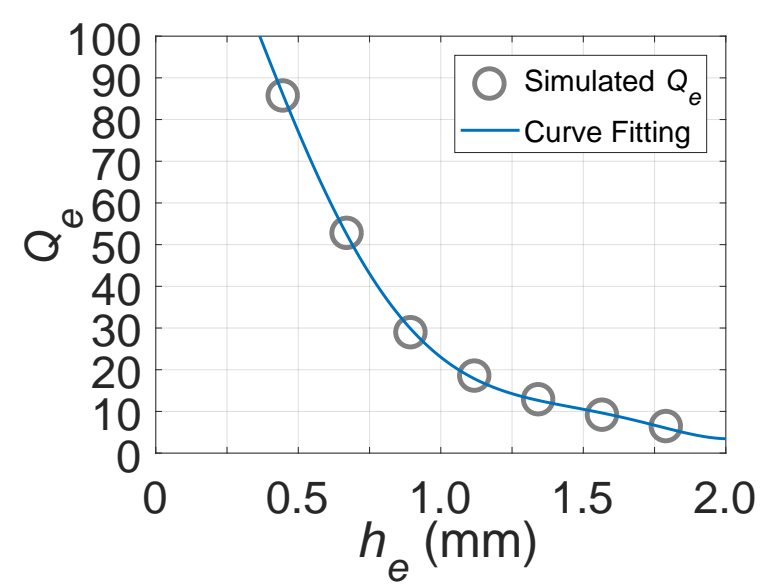

(a)

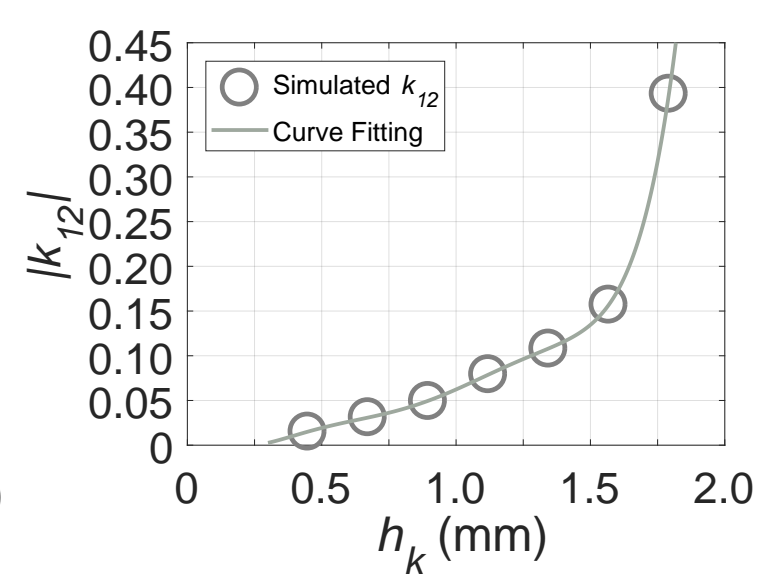

(b)

Figure 6. (a) $Q_{e}$ and (b) $k_{1,2}$ variation versus height of stripline probe in the stack-up expressed as layer number $\left(n_{q e}, n_{k}\right)$.

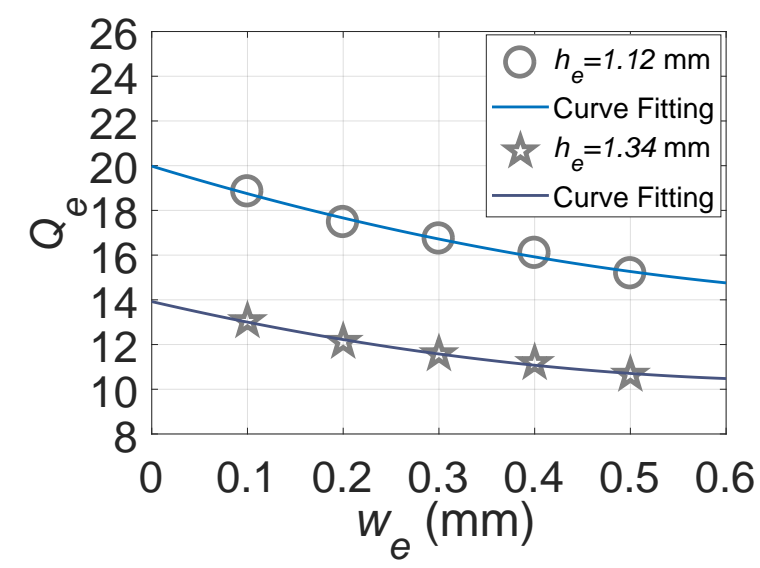

(a)

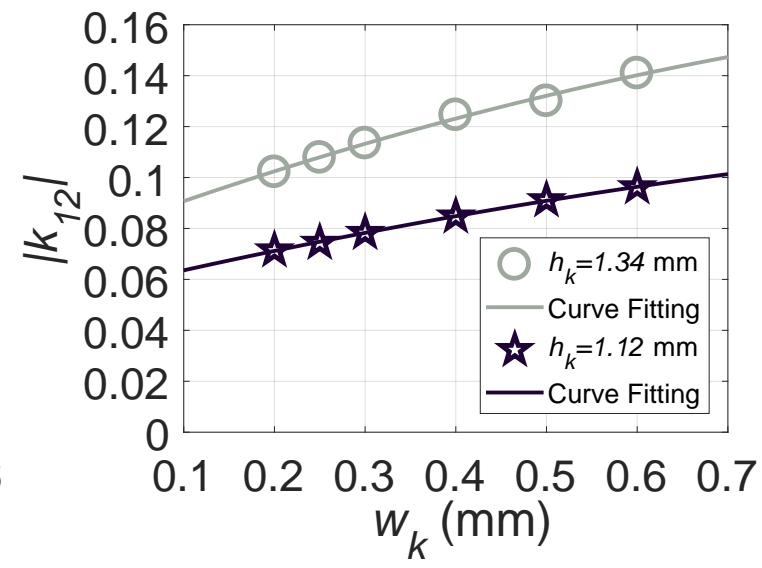

(b)

Figure 7. (a) $Q_{e}$ variation versus stripline probe width $w_{e}$ when $n_{q e}=5$. (b) $k_{1,2}$ variation versus stripline probe width $w_{k}$ when $n_{k}=4$.

\subsection{D EM Simulations of the Filter}

To validate the concept, a CSIW BPF has been designed and simulated. The CSIW resonator previously introduced is the building block of this three-pole BPF whose frequency response will be centered at the L-band. Figure 8a shows the layout of the BPF including its main dimensions. Blue elements highlight the coupling mechanisms, and cover pads that keep an excellent electrical connection among via holes forming the SIW cavity walls on different layers. It should be noted that external and inter-resonator coupling have been accommodated on the very same layer (i.e., $h_{e}=h_{k}=$ $1.12 \mathrm{~mm}$ ) thus allowing for a stack-up simplification and manufacturing cost reduction. Indeed, the designed CSIW components have been included in a multi-project LTCC fabrication run that had an already predefined LTCC stack-up. In this context, the inner conductor printing was only allowed at $h=(1.12,1.792,2.24) \mathrm{mm}$, which limits the filter design flexibility. However, optimized values of the coupling coefficients have been easily obtained by adjusting the probe widths $w_{e}$ and $w_{k}$.

We have chosen to design a direct-coupled three-pole Chebyshev filtering response centered at $1.5 \mathrm{GHz}$ with an equi-ripple bandwidth (BW) of $150 \mathrm{MHz}$, which corresponds to $\mathrm{FBW}=10 \%$ and return loss (RL) of $15 \mathrm{~dB}$. Thus, the coupling coefficients associated with the target response are $Q_{e}=11.19$, and $k_{1,2}=k_{2,3}=0.0879$. Figure $8 \mathrm{~b}$ depicts the simulated filtering response. In the passband, the 
minimum insertion loss (IL) is $1.2 \mathrm{~dB}$ at $1.49 \mathrm{GHz}$, while RL are better than $14.5 \mathrm{~dB}$. Finally, the main dimensions of the filter are detailed in Table 3.

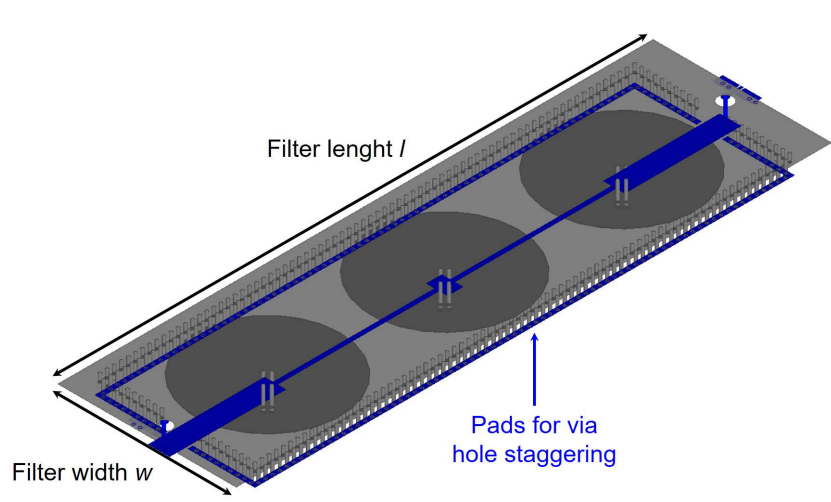

(a)

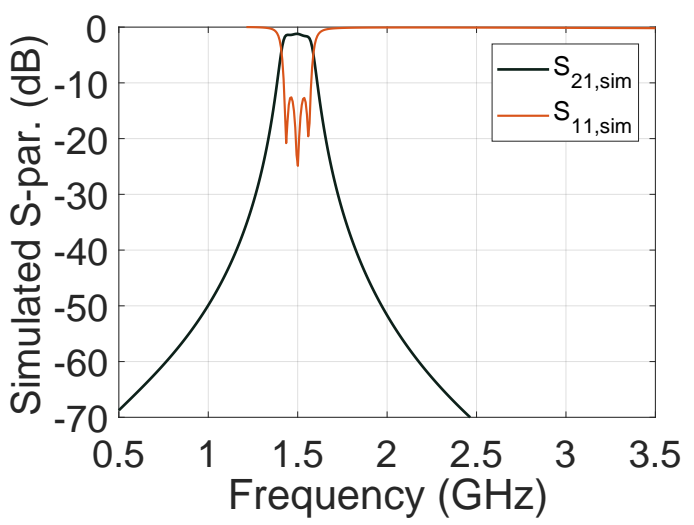

(b)

Figure 8. (a) Three-pole BPF filter layout; (b) Simulated frequency response.

Table 3. Layout Dimensions of the Three-Pole CSIW Filter (Unit: mm).

\begin{tabular}{cccccccccccccc}
\hline $\boldsymbol{l}$ & $\boldsymbol{w}$ & $\boldsymbol{h}$ & $\boldsymbol{d}_{\boldsymbol{v}}$ & $\boldsymbol{d}_{\boldsymbol{h}}$ & $\boldsymbol{r}_{\boldsymbol{p}, \mathbf{1}}$ & $\boldsymbol{r}_{\boldsymbol{p}, \mathbf{2}}$ & $\boldsymbol{h}_{\boldsymbol{p}}$ & $\boldsymbol{g}_{\boldsymbol{p}}$ & $\boldsymbol{w}_{\boldsymbol{e}}$ & $\boldsymbol{h}_{\boldsymbol{e}}$ & $\boldsymbol{w}_{\boldsymbol{k}}$ & $\boldsymbol{h}_{\boldsymbol{k}}$ & $\boldsymbol{p}_{\boldsymbol{d}}$ \\
\hline 32 & 9.5 & 2.24 & 0.68 & 0.18 & 4.3 & 4.36 & 1.792 & 0.448 & 1.25 & 1.12 & 0.25 & 1.12 & 0.4 \\
\hline
\end{tabular}

\section{Results}

As previously mentioned, the DuPont ${ }^{\mathrm{TM}}$ Green Tape ${ }^{\mathrm{TM}}$ 9K7 substrate has been chosen as LTCC dielectric tape in these designs. This is a low-loss glass ceramic dielectric tape for high-frequency applications that includes compatible gold and silver conductors. The final filter stack-up consists of 10 dielectric tape layers with un-fired thickness of $0.254 \mathrm{~mm}$. Then, an additional tape with un-fired thickness of $0.127 \mathrm{~mm}$ has been added on the top of the tenth layer to implement the input-output feeding lines and ground-signal-ground (GSG) probe pads for measuring purposes.

Due to the high number of layers in the stack-up, standard LTCC design rules recommend staggering via holes vertically (i.e., zig-zag position of the holes between layers) to limit the via-posting problem. The latter is a protrusion of the via hole at the external layers to the different $z$-shrinkage of the via fill paste and the tape material, which can provoke metal layer deformations. This technique has been applied in this design as can be seen in Figure 8a.

First, the CSIW resonator presented in Section 2.2 has been fabricated and measured. A photograph of a prototype of the CSIW resonator is shown in Figure 9, while the measured results are shown in Figure 10. The agreement between the simulations and measurements is excellent. The TEM resonant frequency is $f_{r}=1.55 \mathrm{GHz}$, which is just $0.7 \%$ more than the simulated value, while the spurious-free band is around $7.5 \times f_{r}$. In fact, the first high-order mode (i.e., the $\mathrm{TE}_{101}$ ) is centered at $11.6 \mathrm{GHz}$. 


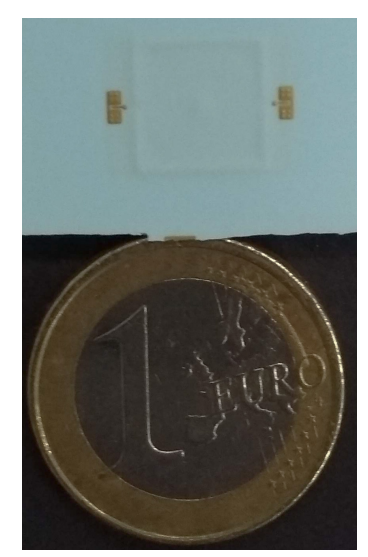

Figure 9. Photo of the CSIW resonator prototype.

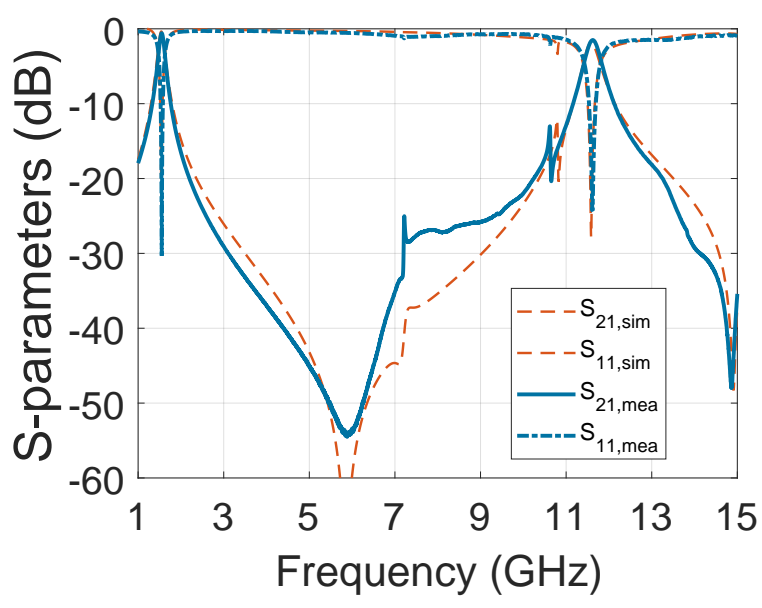

(a)

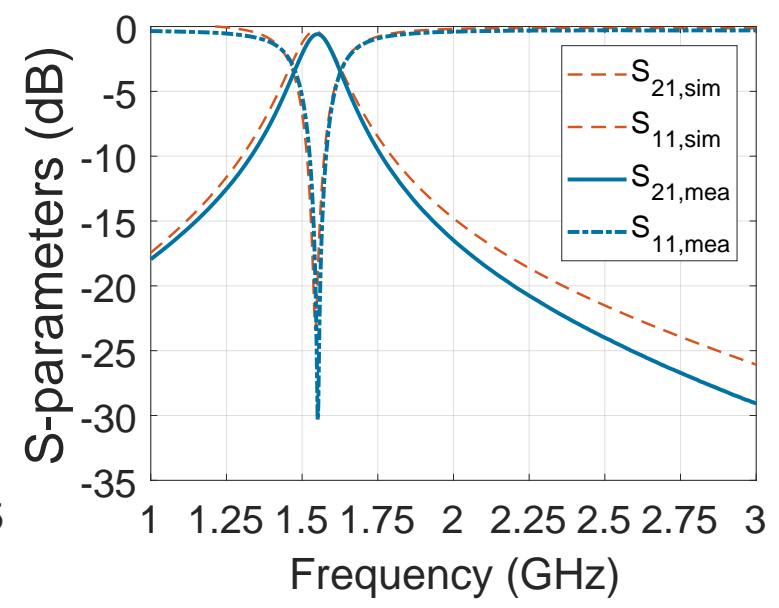

(b)

Figure 10. Measured and simulated results of the embedded coaxial SIW resonator: (a) wideband response up to $15 \mathrm{GHz}$ and (b) passband of the resonance.

In addition, the extracted $Q_{u}$ from measurements is very similar to the simulated one, both being around 150. This value is slightly lower than those represented in Figure 3. Indeed, the CSIW resonators are fabricated in the very same stack-up of the three-pole BPFs, thus they share the same coupling arrangement that results in a more coupled resonators (i.e., measured $S_{21}=-0.6 \mathrm{~dB}$ ).

On the other hand, Figure 11b shows a photograph of an embedded CSIW filter prototype, while the comparison between measured and simulated results are depicted in Figure 12. Again, the agreement between simulations in Ansys HFSS and measurements is excellent. Basically, there is no frequency shift between responses, thus validating the proposed topology and its LTCC manufacturing. The filter IL and RL are $1.2 \mathrm{~dB}$ and around $15 \mathrm{~dB}$, respectively, while the filter FBW is $10 \%$ as expected. The extracted $Q_{u}$ from measurements is close to 165 , thus validating the study of Section 2.2. 


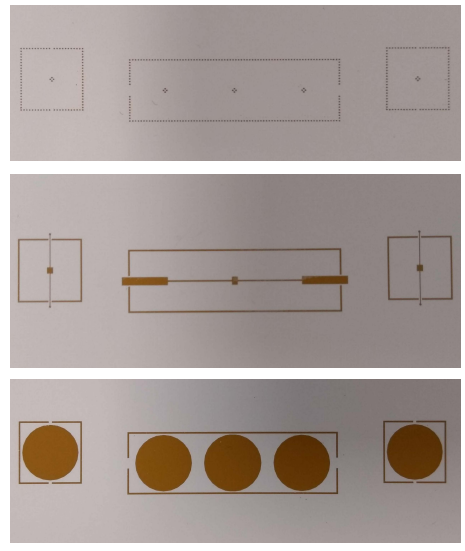

(a)

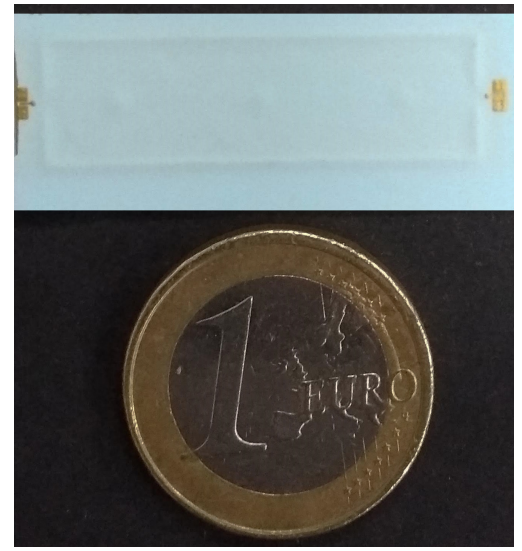

(b)

Figure 11. (a) Photograph of some layers of the LTCC stack-up (i.e., from top to bottom, via holes punching and after printing the coupling and capacitive patch, respectively). The resonator layout is also visible at the sides of the photograph; (b) Photograph of three-pole BPF prototype.

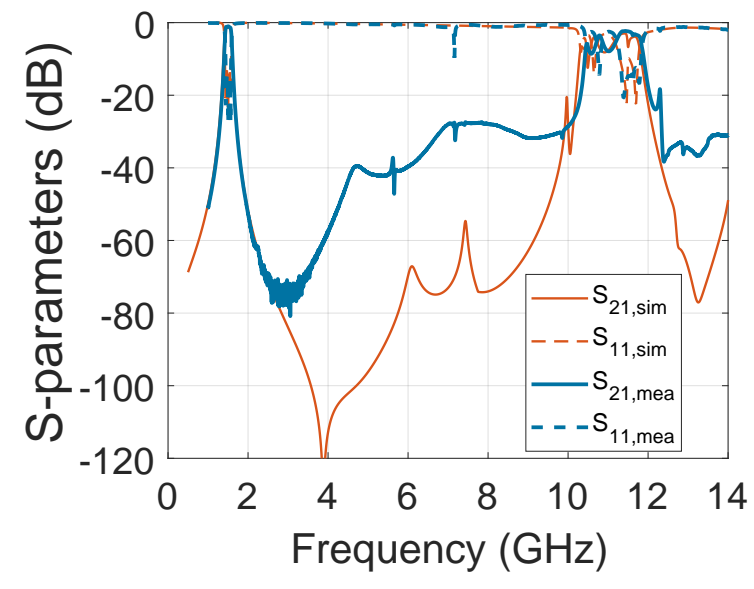

(a)

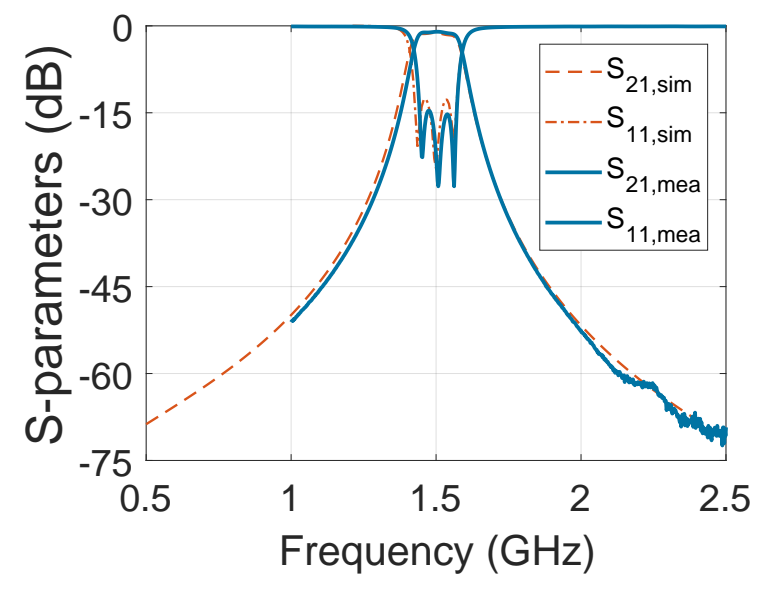

(b)

Figure 12. Measured and simulated results of the three-pole BPF: (a) wideband response and (b) filter passband.

The filter shows a stop-band bandwidth of more than $8 \mathrm{GHz}$, up to $10 \mathrm{GHz}$, with a rejection always better than $30 \mathrm{~dB}$. This means that the spurious-free band is greater than $7 \times f_{0}$, which is very close to the performance of the designed CSIW resonator. Concerning the discrepancy between simulated and measured stop-band performance, this could be due to the probe station setup. Finally, the filter size is $32 \times 9.5 \times 2.24 \mathrm{~mm}^{3}$, which corresponds to $0.426 \times 0.126 \times 0.03 \lambda_{g}^{3}$ and $0.16 \times 0.047 \times 0.01 \lambda_{0}^{2}$, where $\lambda_{g}$ is the guided wavelength at $1.5 \mathrm{GHz}$.

\subsection{Discussion}

To sum up, the designed three-pole filter achieves a MF of $96.8 \%$ while providing a quite wide passband with $\mathrm{FBW}=10 \%$, very wide spurious-free band with a resonator $Q_{u}$ greater than 160 . The latter values confirm the optimal trade-off between remarkable miniaturization design, and wideband filtering response of the proposed magnetic coupling mechanism for embedded CSIW components. In this context, the filter performance in terms of FBW, MF, $Q_{u}$ and spurious-free band of this work are summarized and compared to related references in Table 4.

Wideband filtering responses are extremely complicated to implement in miniaturized SIW BPFs, especially if the MF is strongly increased. For instance, such characteristics have been latterly achieved 
in [8] by using a combination of eight-mode SIW resonators with CRME structures. However, such an approach enables the MF to be higher than $98 \%$ at the cost of a low $Q_{u}$, which has been extracted to be just 90 .

As a comparison, in [5], the authors proposed a three-pole embedded CSIW filter centered at $2.14 \mathrm{GHz}$ with $\mathrm{FBW}=2.8 \%$ whose resonators have an area of $36 \mathrm{~mm}^{2}$, which means that the MF is around $96 \%$. In [18], the designed two-pole and three-pole BPFs based on HMSIW and QMSIW resonators showed passband FBWs of $5.9 \%$ and $4.2 \%$, respectively, at UHF band. However, in the case of the trisection filters, the achieved MF was below $80 \%$.

Finally, some recent works proposed the use of conventional multi-layer PCB technology to implement miniaturized SIW filters based on coaxial topologies. As has been presented in [13], the FBW of the two four-pole CSIW BPFs centered at UHF band is $9.1 \%$ if MF $=92.4 \%$, but is reduced to $4.6 \%$ when the MF is increased up to $96.4 \%$ because standard magnetic post-wall irises were used to couple adjacent CSIW resonators. On the contrary, in [19], third-order BPFs were designed and tested by arranging side-by-side three vertical coaxial stepped-impedance resonators (SIRs), showing an extremely high MF, good $Q_{u}$, but limited spurious-free band.

Table 4. Comparison table between references and proposed CSIW filter.

\begin{tabular}{lccccccc}
\hline Ref. & $f_{\mathbf{0}}(\mathbf{G H z})$ & FBW & Order & $Q_{u}$ & MF (\%) & Area $\mathbf{( m m}^{2}$ ) & Spurious-Free Band \\
\hline$[5,11]$ & 2.14 & $2.8 \%$ & 3 & $\simeq 150$ & $\simeq 96 \%$ & $19 \times 7$ & $>5 \times f_{0}$ \\
[8] & 0.690 & $10.2 \%$ & 2 & 90 & $\simeq 98.8 \%$ & $35 \times 35$ & $4.3 \times f_{0}$ \\
[13] (Figure 4) & 1.66 & $9.1 \%$ & 4 & 276 & $92.4 \%$ & $48 \times 48$ & $6.17 \times f_{0}$ \\
[13] (Figure 6) & 1.04 & $4.6 \%$ & 4 & 248 & $96.4 \%$ & $48 \times 48$ & $3 \times f_{0}$ \\
[18] (Figure 15) & 0.754 & $4.2 \%$ & 3 & $\simeq 190$ & $\simeq 78 \%$ & $41 \times 41.5$ & $>2 \times f_{0}$ \\
[19] (Figure 6) & 3.6 & $6.5 \%$ & 3 & $\simeq 180$ & $\simeq 97 \%$ & $9 \times 12$ & $\simeq 2.5 \times f_{0}$ \\
In this work & 1.5 & $10 \%$ & 3 & $\simeq 165$ & $96.8 \%$ & $32 \times 9.5$ & $7 \times f_{0}$ \\
\hline
\end{tabular}

\section{Conclusions}

In this paper, the design of miniaturized embedded CSIW resonator filters with wideband responses has been investigated. A novel magnetic coupling mechanism has been proposed and studied in detail for both external and inter-resonator couplings. By combining short-ended stripline probes and multi-layer LTCC manufacturing, we can implement extremely compact BPFs with a reasonable level of losses, as well as increase the maximum achievable passband bandwidth in embedded CSIW technology. All concepts have been validated by the simulation and measurement of some fabricated proof-of-concept filtering devices.

Author Contributions: Conceptualization, S.S., J.D.M. and V.E.B.; Funding acquisition, J.D.M. and V.E.B.; Investigation, S.S. and J.D.M.; Methodology, S.S. and J.D.M.; Project administration, J.D.M. and V.E.B.; Resources, S.S.; Supervision, J.D.M. and V.E.B.; Validation, S.S.; Visualization, S.S.; Writing-original draft, S.S.; Writing-review \& editing, J.D.M. and V.E.B.

Funding: This research was funded by Ministerio de Economía, Industria y Competitividad, Spanish Government, under Research Project TEC2016-75934-C4-3-R.

Conflicts of Interest: The authors declare no conflict of interest. The founding sponsors had no role in the design of the study; in the collection, analyses, or interpretation of data; in the writing of the manuscript, and in the decision to publish the results.

\section{Abbreviations}

The following abbreviations are used in this manuscript:

EM Electromagnetic

BPF Bandpass Filter

PCB Printed Circuit Board 


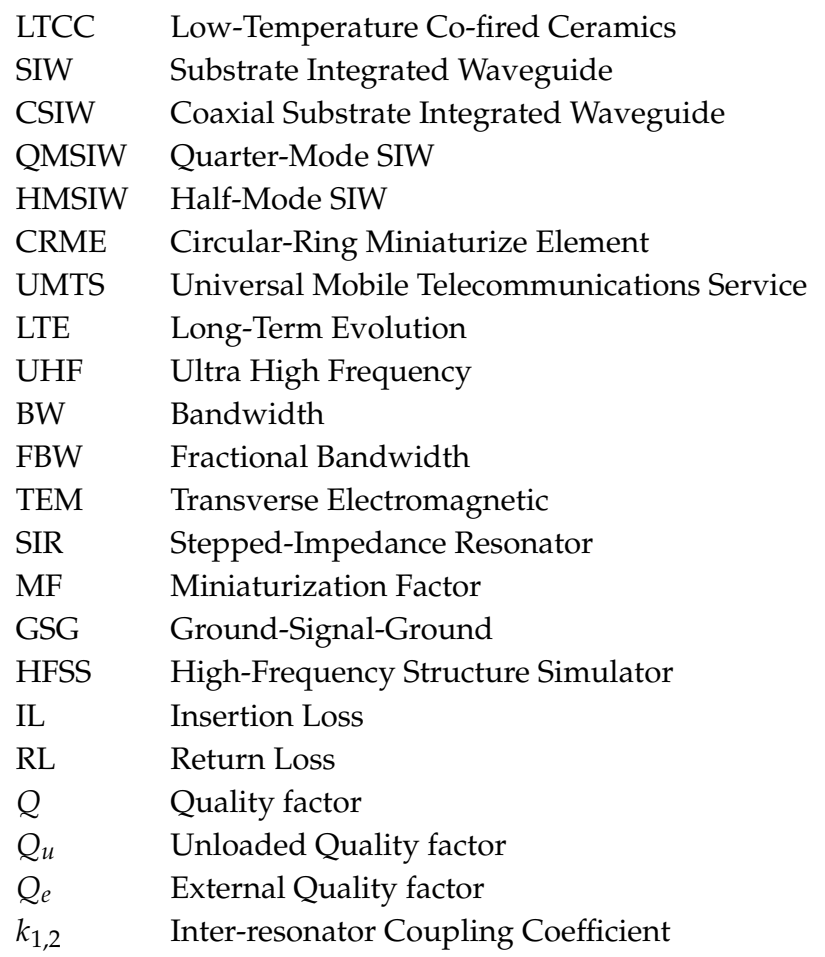

\section{References}

1. Chen, X.P.; Wu, K. Substrate integrated waveguide filters: Practical aspects and design considerations. IEEE Microw. Mag. 2014, 15, 75-83. [CrossRef]

2. Matthaei, G.L. Combline band-pass filters of narrow or moderate bandwidth. Microw. J. 1963, 6, 82-96.

3. Rhodes, J.D. The stepped digital elliptic filter. IEEE Trans. Microw. Theory Technol. 1969, 17, $178-184$. [CrossRef]

4. Hoeft, M.; Ishizaki, T.; Nakakubo, H. Laminated rf Device with Vertical Resonators. Jpn. Patent No. EP 2068393 A1, June 2009.

5. Turgaliev, V.; Kholodnyak, D.; Vendik, I.; Stöpel, D.; Humbla, S.; Müller, J.; Hein, M.A. LTCC highly loaded cavities for the design of single- and dual-band low-loss miniature filters. In Proceedings of the 2010 European Microwave Conference (EUMW), Paris, France, 28-30 September 2010; pp. 180-183.

6. Martínez, J.D.; Taroncher, M.; Boria, V.E. Capacitively loaded resonator for compact substrate integrated waveguide filters. In Proceedings of the 2010 European Microwave Conference (EUMW), Paris, France, 28-30 September 2010; pp. 192-195.

7. Grigoropoulos, N.; Sanz-Izquiredo, B.; Young, P.R. Substrate integrated folded waveguides (SIFW) and filters. IEEE Microw. Wirel. Compon. Lett. 2005, 15, 829-831. [CrossRef]

8. Li, L.; Wu, Z.; Yang, K.; Lai, X.; Lei, Z. A novel miniature single-layer eighth-mode SIW filter with improved out-of-band rejection. IEEE Microw. Wirel. Compon. Lett. 2018, 28, 407-409. [CrossRef]

9. Martínez, J. D.; Sirci, S.; Taroncher, M.; Boria,V.E. Compact CPW-fed combline filter in substrate integrated waveguide technology. IEEE Microw. Wirel. Compon. Lett. 2012, 22, 7-9. [CrossRef]

10. Kholodnyak, D.; Turgaliev, V.; Vorobev, E. Advanced microwave filters in LTCC and PCB technologies. In Proceedings of the 2014 Asia-Pacific Microwave Conference (APMC), Sendai, Japan, 4-7 November 2014; pp. 88-90.

11. Turgaliev, V.; Kholodnyak, D.; Müller, J.; Hein, M.A. Small-size low-loss bandpass filters on substrate-integrated waveguide capacitively loaded cavities embedded in low temperature co-fired ceramics. J. Ceram. Sci. Technol. 2015, 6, 305-314.

12. Nassar, S.O.; Meyer, P. Pedestal substrate integrated waveguide resonators and filters. IET Microw. Antennas Propag. 2017, 11, 804-810. [CrossRef]

13. Ho, M.H.; Li, J.C.; Chen, Y.C. Miniaturized SIW cavity resonator and its application in filter design. IEEE Microw. Wirel. Compon. Lett. 2018, 28, 651-653. [CrossRef] 
14. Sirci, S.; Sánchez-Soriano, M.Á.; Martínez, J.D.; Boria, V.E.; Gentili, F.; Bösch, W.; Sorrentino, R. Design and multiphysics analysis of direct and cross-coupled SIW combline filters using electric and magnetic couplings. IEEE Trans. Microw. Theory Technol., 2015, 63, 4341-4354. [CrossRef]

15. Hong, J.S. Microstrip Filter for RF/Microwave Applications; 2nd ed.; John Wiley and Sons, Inc.: Hoboken, NJ, USA, 2011.

16. Riblet, H.J. An accurate approximation of the impedance of a circular cylinder concentric with an external square tube. IEEE Trans. Microw. Theory Technol. 1983, 31, 841-844. [CrossRef]

17. Landau, L.D.; Lifschitz, E.M. Electrodynamics of Continuous Media (Volume 8). In A Course of Theoretical Physics; Permagon Press: Oxford, UK, 1987.

18. Pourghorban Saghati, A.; Pourghorban Saghati, A.; Entesari, K. Ultra-miniature SIW cavity resonators and filters.IEEE Trans. Microw. Theory Technol. 2015, 63, 4329-4340. [CrossRef]

19. Yang, T.; Ho, K.; Rebeiz, G.M. Compact self-shielded 2-3 GHz high-Q coaxial fixed and tunable filters. IEEE Trans. Microw. Theory Technol. 2014, 62, 3370-3379. [CrossRef]

(C) 2019 by the authors. Licensee MDPI, Basel, Switzerland. This article is an open access article distributed under the terms and conditions of the Creative Commons Attribution (CC BY) license (http:/ / creativecommons.org/licenses/by/4.0/). 